\title{
Writing about the Personal Utility of Learning Contents in a Learning Journal Improves Learning Motivation and Comprehension
}

\author{
Kristin Schmidt, Julia Maier, and Matthias Nückles \\ Department of Educational Science, University of Freiburg, Rempart Straße 11, D-79098 Freiburg, Germany
}

Correspondence should be addressed to Kristin Schmidt, kristin.schmidt@ezw.uni-freiburg.de

Received 30 May 2012; Revised 17 September 2012; Accepted 8 November 2012

Academic Editor: Susanne Narciss

Copyright ( $\odot 2012$ Kristin Schmidt et al. This is an open access article distributed under the Creative Commons Attribution License, which permits unrestricted use, distribution, and reproduction in any medium, provided the original work is properly cited.

Reflecting on the personal utility and value of learning contents is important for motivation building and engagement in high quality learning processes. We investigated the effects of a personal-utility prompt in journal writing on students' learning motivation and comprehension in biology education. 40 students of a German secondary school took part in a quasi-experimental field study. The students kept a weekly learning journal over six weeks. For writing their journal entries, the students received a brief instruction that either did or did not include a personal-utility prompt. Results showed that the personal-utility prompt successfully supported the students in reflecting about the personal utility of the learning contents. Consequently, students in the personal-utility prompt condition reported higher degrees of learning motivation and achieved better comprehension scores as compared to students who had no personal-utility prompt available. Evidently, using journal writing to reflect upon the utility and value of learning contents is a beneficial method to support students' learning motivation and comprehension in secondary science education.

\section{Introduction}

The ability to self-regulate one's learning processes effectively is important at almost all levels of education. Self-regulated learning involves the ability to use cognitive, metacognitive, and motivational learning strategies effectively [1]. One important motivational learning strategy a self-regulated learner should possess is the ability to explain to oneself why it is worthwhile to learn a particular topic and what the personal utility of a topic could be [2]. Far too often, however, students find it difficult to see the personal utility of the contents discussed, especially in secondary science education, for example [3]. Consequently, students show little identification with the learning contents and are weakly motivated to invest substantial effort in high quality learning processes [4]. On the contrary, they seem to limit their effort to the minimum defined by classroom requirements. As a result, students tend to use superficial learning strategies and accordingly focus on rehearsing and memorizing the contents as "isolated facts" [5]. Therefore, deep understanding and long-time retention can hardly be achieved because the students fail to engage in intentional and meaningful learning [6].

Learning journals are a medium which can help students reflect on the relevance of a particular topic. In a learning journal, students typically write down their thoughts on previously presented learning contents. They try to articulate what they found personally interesting and important and how the new information relates to what they already know about the subject. The aim of the current study was to investigate whether the reflection about the relevance of a topic can increase students' learning motivation and their comprehension of the subject matter.

Reflective journal writing can be regarded as a medium to foster self-regulated learning [7]. Journal writing promotes deep processing of the learning contents as students are encouraged to apply cognitive as well as metacognitive and 
motivational learning strategies [7-10]. In contrast to essays or scientific articles, learning journals do not have a fixed rhetorical structure. Therefore, they are especially beneficial for learners with comparatively little writing expertise [7]. Learning journal instructions are used to encourage students to use beneficial learning strategies via prompting. Prompts are strategy activators in the form of questions or hints that trigger students' application of corresponding strategies [11]. Previous research provided ample evidence that journal writing can be an effective medium to promote self-regulated learning. Positive effects of journal writing on learning behavior and learning outcomes were found both in lab and field studies in such diverse domains as biology, math, and psychology ([7-9, 12-14]; see [10] for an overview). Berthold et al. [8] and Nückles et al. [7], for example, investigated the effects of cognitive and metacognitive prompts on the application of learning strategies in journal writing as well as on learning outcomes. The results of their studies showed that prompting a combination of cognitive and metacognitive learning strategies in journal writing resulted in large effects on learning outcomes with regard to the acquisition of deep comprehension and retention of the acquired knowledge (see [13]). However, despite the promising short-term results of cognitive and metacognitive prompts in those experimental studies, Nückles et al. [15] also found a decrease in students' learning motivation over a longer period of time that was associated with a decrease in learning outcomes. Thus, prompting cognitive and metacognitive strategies in journal writing apparently was not sufficient for maintaining effort and interest in the learning contents over a longer period of time. Theoretically, the regulation of motivation is regarded as an essential subprocess in current models of self-regulated learning as well $[16,17]$. Hence, prompting motivational strategies, which was not realized in the above-mentioned studies, seems to be equally as important as cognitive and metacognitive strategies. Thus, it is an interesting and open question whether the prompting of motivational strategies in journal writing will promote learning motivation. Accordingly, in our present study on journal writing, we introduced and systematically varied a motivational prompt - in addition to a combination of cognitive and metacognitive prompts. In the following, we discuss in a theoretical context the role of motivation in self-regulated learning and how learning journals can support the regulation of motivation and thereby learning successfully.

To describe the processes involved in self-regulated learning and the interrelations between different components, several models of self-regulated learning have been suggested (e.g., [17-20]). The perhaps most well-known one is the model developed by Zimmerman [20], which describes self-regulated learning as a cyclical and interactive process. According to this model, the coordination and regulation of cognition is realized by the metacognitive strategies of planning, monitoring, and evaluation of the learning process [1]. Furthermore, the learner should employ motivational strategies to initiate the learning process, to shield it against interruptions, and to invest a sustained effort in meaningful cognitive learning activities [16]. In order to maintain learning motivation, it is particularly important that learners experience the learning tasks and topics as personally valuable [21]. The more the learners perceive the learning contents as personally relevant, useful, and interesting [22], the more they will engage in effortful and persistent intentional learning [6, 22-24]. For example, by reflecting and writing about the relevance of a wellfunctioning immune system, learners could learn to regard this topic as also being relevant for their own health. As a consequence, they would more easily identify with the learning contents. They would develop a desire to acquire as much knowledge about the topic as possible and explore it for their own interest. Accordingly, we provided the students in our study with a personal-utility prompt in order to help them discover and articulate the personal relevance of the learning contents by writing a learning journal. In doing so, we expected to improve students' motivation to engage in meaningful leaning.

Prior research on motivation and learning provided evidence that students with higher learning motivation tend to choose more challenging learning goals $[25,26]$, apply more self-regulatory strategies [27], and show greater strategic flexibility [27, 28], more meaningful cognitive engagement $[29,30]$, and greater academic achievement $[30,31]$. However, students also seem to have problems in motivating themselves [32]. Especially novices in a discipline may find it difficult to motivate themselves [16] and therefore are at risk to lose their learning motivation [32]. One strategy to increase students' learning motivation is to help them perceive the value and personal relevance of a topic $[1,17,33]$. In this regard, learning journals seem to be a particularly promising medium because they offer students ample freedom for reflecting on the meaning and purpose of a particular topic.

Such reflection is especially important in science education, as students are expected not only to acquire factual knowledge but also a thorough conceptual understanding of the subject matter [34]. In this regard, previous research in biology education demonstrated the benefits of learning journals [9, 12]. McCrindle and Christensen, for example, compared reflective journal writing with the writing of a scientific report in undergraduate biology courses. They found that - in comparison with the students in the scientific report condition-students who wrote regular learning journal entries as a follow-up course work reported using more sophisticated cognitive and metacognitive learning strategies and showed a more complex and better integrated understanding in a test at the end of the course. Such an integrated and flexible understanding is necessary to successfully apply the acquired knowledge to real-life situations and to participate in social discourses about science and the role of science in society [2]. For this reason, it is important that students engage in exploring the meaning and value of the contents discussed in science classes at school. Learning journals offer an ample opportunity for such reflection. For example, students who reflect on the meaning and purposes of immunology in a biology class 
could learn to consider this knowledge as valuable because it helps to prevent illness or to understand the signals of the body and medical advice. In this way, journal writing in science education may contribute to increases in students' learning motivation and improve their learning behavior in science classes, such as in biology.

\section{Research Questions and Hypotheses}

Based on these theoretical considerations, we addressed the following research questions. First of all, we were interested in whether a personal-utility prompt would lead the students to reflect about the meaning, purpose, and relevance of the learning contents in the learning journals. Second, related to this research question, we tested whether the personal-utility prompt would improve students' motivation for learning biology as well as their learning outcomes as measured by a comprehension test.

Accordingly, we predicted that students who received a personal-utility prompt would show a greater amount of statements about the meaning, purposes, and relevance of a topic in their learning journals as compared with students who did not receive such a prompt (Hypothesis 1). Writing about the relevance of a topic could help to make the value of a topic explicit and in this way increase students' learning motivation. As higher learning motivation should entail more effortful and persistent engagement in the exploration of a topic, we expected that providing students with a personal-utility prompt for journal writing would also have a positive effect on learning outcomes, that is, comprehension of the topic. Thus, we predicted that students who received a personal-utility prompt would report higher levels of motivation after journal writing (Hypothesis 2) and also show higher scores in a comprehension test as compared with students who wrote their learning journals without this prompt (Hypothesis 3 ).

\section{Method}

3.1. Participants and Design. 40 high school students (seventh grade, 13-14 years old) participated in the quasiexperimental field study. They were members of two biology classes of a German secondary school and taught by the same biology teacher. During the surveyed timespan of six weeks, the students reflected on the learning contents of their biology lessons by writing regular learning journal entries. The lessons were about basic concepts and issues in immunology (e.g., the functioning of white blood cells or the functioning of the Human Immunodeficiency Virus). Students in both classes were asked to write a learning journal entry once a week, that is, six entries in total. As the students' accomplishment of the journal writing assignment was not checked by the teacher, about one-third of the students in both classes ( 23 out of $N=63$ ) did not comply with the assignment and did not write any learning journal entries. We therefore excluded these students from the data analyses. To support the students' journal writing, we provided them with a combination of cognitive and metacognitive prompts that had repeatedly been shown to foster learning processes and learning outcomes in our previous research (see $[7$, $13,15]$ ). We used a one factorial between-subject design comprised of two experimental conditions. The students of one class were assigned to the experimental condition while the students of the other class were assigned to the control condition. Given that the students belonging to a particular class as a whole were assigned to either the experimental or control condition, random assignment was somewhat restricted. Therefore, our design was rather quasi experimental than experimental in a strict sense. To nevertheless keep both conditions as comparable as possible, the same teacher taught the same contents in both classes during the intervention. She also used the same didactic methods and learning materials in both classes. All participating students had received biology instruction for two years. Their prior knowledge and also their motivation in learning biology were comparable across conditions (for details on statistical tests regarding prior knowledge and motivation prior to the journal writing intervention, see Section 4).

The students in the experimental condition received a personal-utility prompt in addition to our standard combination of cognitive and metacognitive prompts (personalutility prompt condition, $N=19,7$ boys, 12 girls). The students in the control condition received the same combination of cognitive and metacognitive prompts but no additional personal-utility prompt (standard prompts condition, $N=21$, eleven boys, ten girls). The distribution of boys and girls did not differ significantly between the experimental conditions, $\chi^{2}(N=40)=0.97$, ns. On average, students who complied with the journal writing task wrote 4.63 ( $\mathrm{SD}=0.98)$ out of 6 possible learning journal entries. Dependent variables encompassed students' comprehension of biological concepts as well as their learning motivation assessed by a learning motivation questionnaire. In addition, we analyzed measures of learning strategies elicited in the learning journals.

\subsection{Instruments and Coding}

3.2.1. Measures of Acceptance. To assess the students' acceptance of journal writing, we used 10 items translated and adapted from the Intrinsic Motivation Inventory (IMI; [35]). The IMI is a multidimensional questionnaire. The items that we chose assessed the students' interest and enjoyment (e.g., "journal writing was fun for me"), their effort (e.g., "I spent much effort in journal writing"), and perceived usefulness of the performed task (e.g., "journal writing was helpful for better understanding of the learning contents" (see Appendix A for the complete sample of items). Students estimated their degree of agreement for each item on a 7-point rating scale ranging from 1 (very low degree of agreement) to 7 (very high degree of agreement). Based on the students' answers to the items, we computed an average score of journal writing acceptance for each student. The internal consistency measured by the Cronbach's alpha was very good, $\alpha=.93$. 
3.2.2. Measures of Learning Motivation. To assess the students' learning motivation related to biology, we used adapted items for interest (e.g., "I enjoyed tricky tasks and puzzles in biology very much."), effort (e.g., "I tried hard while solving problems in biology."), and perceived competence (e.g., "I could solve my tasks pretty well in biology.") from the Intrinsic Motivation Inventory (IMI; [35]) related to biology tasks. In total, the questionnaire included 18 self-report items to be rated on a 7-point rating scale, ranging from 1 (very low degree of agreement) to 7 (very high degree of agreement). As the internal consistency measured by Cronbach's alpha was good, Cronbach's $\alpha=$ 0.79 , we computed an average motivation score. High motivation scores indicated self-efficient students that were interested in the topic and willing to invest effort in biology tasks. We assessed students' learning motivation related to biology topics before and after the period of journal writing.

3.2.3. Comprehension Test. To assess the students' comprehension of immunology, we designed a comprehension test based on the guidelines for the biology curriculum of German high schools (see Appendix B for the test items). To ensure validity, the test was assessed by two experienced teachers of biology. We asked the students to answer the five questions in the comprehension test before and once again after the 6-week period of journal writing. Between these points of measurement, the teacher instructed both classes in immunology using the same teaching methods and materials. As the guidelines in the curriculum focus on the ability to explain biological phenomena scientifically, we primarily designed explanation tasks that measured comprehension (see Appendix B). Thus, the students had to apply their acquired knowledge in order to generate explanations. Recalling facts would not have been enough to answer the questions appropriately. Two trained research assistants evaluated each question of the comprehension test. They compared students' answers with reference answers given by the teacher and counted the number of correct statements. A maximum of 22 points could be reached when answering the five test items completely correct. The maximum scores, as well as the means and standard deviations students reached on average on each item in the pre- and posttest, are presented in Appendix B. Interrater reliability as determined by Cohen's kappa was very good, $\kappa=0.95$. Students reached an average comprehension score of $\mathrm{M}=5.07(\mathrm{SD}=1.86)$ in the pretest and $\mathrm{M}=11.47$ $(\mathrm{SD}=3.28)$ in the posttest.

3.2.4. Writing Assignment. As the participating 7th-grade students were inexperienced in journal writing, we provided them with a brief instruction (300 words) on how to write a learning journal. Students in both experimental groups were asked to reflect on the topics discussed in class by writing about the most important contents of a lesson, about their own understanding of concepts, and questions that still remained open to them after class discussion. The students were told to write about one page per journal entry. To facilitate the journal writing, the instruction
TABLE 1: Prompts used in the writing instructions.

Prompts

Cognitive prompts (organization and elaboration)

How can you structure and summarize the contents in a meaningful way?

Which examples can you think of that illustrate, confirm, or conflict with the learning contents?

Metacognitive prompts (monitoring and planning of remedial strategies)

Which main points have you understood yet, and which points do you need to elaborate?

What possibilities do you have to overcome the comprehension problems?

Personal-utility prompt

Why is the learning material personally relevant for you at present or in future out of school?

Note. Students in the standard prompts condition received only the cognitive and metacognitive prompts. Students in the personal-utility prompt condition additionally received the personal-utility prompt.

included two cognitive prompts stimulating elaboration and organization strategies and two metacognitive prompts stimulating monitoring and planning of remedial strategies (see Table 1). Students in the personal-utility prompt condition additionally received a personal-utility prompt that asked them to think about the personal relevance of the topic. The instruction ended by encouraging the students to consider personal needs, to develop their own ideas, and to design their learning journal however they wished.

3.2.5. Coding of the Learning Journals. Apart from the preand posttests, we also assessed the students' learning processes and strategies elicited in the learning journals. For this analysis, we used the coding scheme developed by Nückles and colleagues [7], see also Glogger et al. [12]. We aimed to identify learning strategies triggered by the corresponding prompts. Two independent raters, who were blind to the experimental conditions, coded the learning journals on the granular level of individual statements. As preparation for the coding, we first segmented the texts into single statements. We split longer sentences into smaller units on the basis of grammatical and organizational markers (e.g., and, or, because, etc.; see [36]). Based on this segmentation, the raters categorized single statements as organization, elaboration, metacognition, or personal-utility statements. For example, statements that highlighted the main points of the topic and their interrelations were coded as indicators of organization (e.g., students underlined important terms or highlighted them with different colors). As elaboration, we coded statements in which students associated the new content with their prior knowledge, for example, by generating examples, analogies, or illustrations (e.g., "the human immune system can be compared with the protective walls of a castle."). We coded statements related to comprehension 
monitoring (e.g., "I have difficulties in understanding the differences in active and passive immunizations.") and planning of remedial strategies (e.g., "I will rework the course materials and ask the teacher if I cannot understand everything.") as metacognitive strategies. Finally, we analyzed to what extent the students articulated considerations regarding the personal relevance and importance of the topic or their own interest in the topic (e.g., "it is important for me to know how I can prevent the transmission of HIV infection."). We coded these statements as personalutility statements. Interrater reliabilities as determined by Cohen's kappa were very good (between $\kappa=0.84$ and $\kappa=$ $0.93)$.

3.3. Procedure. The whole intervention lasted eight weeks. In the first week, we asked the students to take part in a pretest. The students took the pretest in their class. They estimated their motivation in learning biology by using the learning motivation questionnaire described above. To assess the students' prior knowledge in immunology, they tried to answer the questions of the comprehension test (see Appendix B). Afterwards, they were given the abovedescribed brief instruction on why and how to write a learning journal. The instruction was handed out to the students on a sheet of paper and was also orally explained to them by a preservice teacher, who served as the experimenter in this study. The instruction was identical for all students except for the personal-utility prompt. The students in both experimental conditions received two cognitive and two metacognitive prompts. However, the students in the personal-utility condition additionally received a personalutility prompt. The students read the instruction together with the experimenter, who emphasized the relevance of using the prompts. The students were asked to write a learning journal entry once a week after the two biology lessons that were taught en bloc. The students were asked to show their biology teacher the journal entries but they received no feedback by the teacher. We decided against providing feedback on the journals to keep the implementation of the intervention as objective and comparable as possible across individual students. The students wrote a maximum of 6 journal entries during the intervention period. The students were asked to put the learning journal entries together into a small booklet, which was collected by the experimenter at the end of the journal writing period prior to the posttest. In the last week, all students took part in the posttest in class. The students again assessed their motivation in learning biology using the same questionnaire as in the pretest. They answered the explanation questions of the comprehension test and, finally, rated their acceptance of journal writing using the acceptance questionnaire mentioned above. All tests were administered as paper-pencil tests. Students completed the tests in their regular biology lesson in week eight of the overall intervention. After the end of the study, the students in the control condition also received the instruction with the personal-utility prompt to enable them to benefit as well from this enhanced instruction when writing future learning journals.
TABLe 2: Means and standard deviations for the experimental conditions.

\begin{tabular}{|c|c|c|c|c|}
\hline \multirow{3}{*}{ Measures } & \multicolumn{4}{|c|}{ Experimental condition } \\
\hline & \multicolumn{2}{|c|}{$\begin{array}{l}\text { Personal-utility } \\
\text { prompt condition }\end{array}$} & \multicolumn{2}{|c|}{$\begin{array}{c}\text { Standard prompts } \\
\text { condition }\end{array}$} \\
\hline & $\mathrm{M}$ & $(\mathrm{SD})$ & $\mathrm{M}$ & $(\mathrm{SD})$ \\
\hline \multicolumn{5}{|l|}{ Pretest } \\
\hline Comprehension $^{\mathrm{a}}$ & 5.00 & $(2.05)$ & 5.13 & $(1.73)$ \\
\hline Learning motivation ${ }^{\mathrm{b}}$ & 4.92 & $(0.46)$ & 4.66 & $(0.49)$ \\
\hline \multicolumn{5}{|l|}{ Elicited strategies ${ }^{c}$} \\
\hline Elaboration & 0.51 & $(0.18)$ & 0.46 & $(0.16)$ \\
\hline Organization & 2.48 & $(1.21)$ & 2.66 & $(1.22)$ \\
\hline Metacognitive strategies & 0.25 & $(0.21)$ & 0.22 & $(0.16)$ \\
\hline Personal-utility statements & 0.54 & $(0.61)$ & 0.08 & $(0.21)$ \\
\hline \multicolumn{5}{|l|}{ Posttest } \\
\hline Comprehension $^{\mathrm{a}}$ & 13.11 & $(2.81)$ & 10.00 & $(3.00)$ \\
\hline Learning motivation ${ }^{\mathrm{b}}$ & 5.11 & $(0.49)$ & 4.42 & $(0.64)$ \\
\hline Acceptance $^{\mathrm{b}}$ & 5.61 & $(1.09)$ & 4.68 & $(1.34)$ \\
\hline
\end{tabular}

Note. The different numbers of students in pre- and posttest were owing

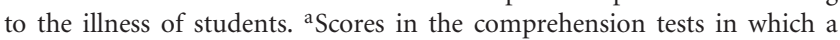
maximum of 22 points was achievable. ${ }^{b}$ Rating scores from 1 (low value) to 7 (high value). ${ }^{c}$ Average number of strategy indicators elicited in one learning journal.

\section{Results}

Table 2 presents the mean scores and standard deviations separately for the experimental conditions and thus provides an overview of the variables of interest. In the following sections, we first analyzed the pretest scores, then the measures of the learning strategies elicited in the learning journals, and finally the posttest scores. As an effect size measure for group differences, we used partial $\eta^{2}$ qualifying values $<0.06$ as small effect, values in the range between 0.06 and 0.13 as medium effect, and values $>0.13$ as large effect (see [37]).

4.1. Investigating Pretest Scores. First, we investigated whether students in the experimental conditions had similar prior knowledge related to immunology as well as comparable learning motivation. We conducted a one-factorial analysis of variance with prior knowledge as dependent variable and treatment condition (personal-utility prompt condition versus standard prompts condition) as independent variable. The results indicated no significant differences for the experimental conditions regarding prior knowledge, $F(1,35)=$ 0.04 , ns. (Small differences regarding the degrees of freedom are due to the fact that three students missed the prior knowledge test and four students failed to complete the motivation questionnaire in pretest session. In the posttest, two students were ill and failed to complete both the comprehension test and motivation questionnaire.) An analysis of variance with the motivation scores as the dependent and treatment condition as the independent variable was also not significant, $F(1,34)=1.00$, ns. Thus, the students in both experimental conditions had comparable prior knowledge 
and also comparable motivation in learning biology prior to our treatment.

\subsubsection{Hypothesis 1: Personal-Utility Prompt Stimulates Reflec-} tions about the Personal Utility of Immunology in the Learning Journals. First, we investigated whether the students in the personal-utility prompt condition produced more statements about the meaning, purpose, and personal relevance of immunology than the students in the standard prompts condition. To test this hypothesis, we conducted a onefactorial analysis of variance with the two experimental conditions (personal-utility prompt condition versus standard prompts condition) as the independent and the number of personal-utility statements as the dependent variable. The results indicated that the treatment was successful, $F(1,38)=$ $10.42, p<0.01$, partial $\eta^{2}=0.21$. Students in the personalutility prompt condition produced significantly more statements regarding the personal relevance of immunology in their learning journals than the students in the standard prompts condition. Indeed, the students in the standard prompts condition hardly articulated any thoughts regarding the utility and personal value the topic of immunology had for them (see Table 2).

As both treatment groups received the same cognitive and metacognitive prompts, we did not expect any significant differences in cognitive and metacognitive strategy use between the groups. Inline with this assumption, a multivariate analysis of variance with the frequencies of elaboration, organization, and metacognitive strategies as dependent variables and experimental condition as independent variable clearly failed to reach statistical significance, Pillai's trace $=.03, F(3,36)=0.37$, ns. Table 2 shows that the students in both conditions produced only a few metacognitive statements indicating monitoring or planning of remedial strategies (around one statement in four journal entries). The application of elaboration strategies was also low (around one statement in two journal entries). In contrast, the use of organizational strategies was relatively high ( 2.5 statements per journal entry). To investigate these differences in strategy use in more detail, we conducted a repeated measures analysis of variance with the different kinds of strategies as a within-subjects factor. This MANOVA indicated significant differences between the frequencies of students' strategy use, Pillai's trace $=0.84, F(2,37)=96.04$, $p<0.01$, partial $\eta^{2}=0.84$. Pairwise comparisons showed that the students used significantly more organization strategies than elaboration strategies, $F(1,39)=104.87, p<0.01$ partial $\eta^{2}=0.73$, and significantly more elaboration strategies than metacognitive strategies, $F(1,39)=60.37, p<0.01$ partial $\eta^{2}=0.61$. Overall, the application of cognitive and metacognitive strategies in the learning journals was rather low, with the exception of organization strategies.

\subsubsection{Hypothesis 2: Reflecting about the Personal Utility} of the Topic Immunology Increases Students' Motivation in Learning Biology. To test this hypothesis, we computed an analysis of variance with the posttest motivation scores as the dependent and the experimental condition (personal-utility prompt condition versus standard prompts condition) as the independent variable. The pretest motivation scores were included as a covariate. The ANCOVA showed a significant effect of the pretest on the posttest scores, $F(1,32)=14.55$, $p<0.01$, partial $\eta^{2}=0.31$, indicating the relative stability of individual differences in students' motivation in learning biology. Nevertheless, as predicted, the students in the personal-utility prompt condition had significantly higher motivation posttest scores than the students in the standard prompts condition, $F(1,32)=14.76, p<0.01$, partial $\eta^{2}=0.32$. Thus, irrespective of the apparent interindividual differences in students' motivation in biology, reflecting about the personal utility of the topic of immunology through journal writing evidently helped the students to substantially increase their motivation for learning biology.

We further conducted an analysis of variance to test for differences between the experimental conditions regarding the students' acceptance of journal writing. The ANOVA indeed proved to be significant, $F(1,36)=5.44, p<0.05$, partial $\eta^{2}=0.13$. Students who had received a personalutility prompt were more likely to perceive journal writing as a beneficial learning method that they would like to use in future learning than students who had received only cognitive and metacognitive prompts.

\subsubsection{Hypothesis 3: Reflecting about the Personal Utility} of Immunology Increases Students' Comprehension of this Topic. To investigate the students' comprehension regarding immunological concepts, we conducted an analysis of variance with the individual posttest comprehension scores as the dependent variable and the experimental condition (personal-utility prompt condition versus standard prompts condition) as the independent variable. The pretest comprehension scores were included as a covariate. The results indicated a significant effect of the pretest on the posttest scores, $F(1,33)=6.59, p<0.05$, partial $\eta^{2}=0.17$. Over and above these interindividual differences, the students in the personal-utility prompt condition evidently achieved higher scores in the comprehension posttest than the students in the standard prompts condition, $F(1,33)=11.93, p<0.01$, partial $\eta^{2}=0.27$. Thus, reflecting about the personal utility of the topic of immunology in a learning journal not only increased the students' motivation for learning biology, but also spurred them to achieve superior comprehension than the students in the standard prompts condition.

To explore this result in more detail, we conducted a multiple regression analysis (backward method) to identify predictors of posttest comprehension scores. As potential predictors, we included the frequency of personal-utility statements as well as statements indicating elaboration, organization, and metacognitive strategies. The pretest comprehension scores and the posttest motivation scores were also included. The only predictor of posttest comprehension that proved to be significant was the posttest motivation score, $\beta=0.38, p<0.05 ; R^{2}=0.14$. In the next step, we investigated whether the posttest motivation score could be predicted by the frequency of statements representing different types of strategies (personal utility, organization, 
elaboration, and metacognition) elicited in the learning journals. Accordingly, we conducted a multiple regression analysis with the posttest motivation scores as criterion and the frequency of personal utility, elaboration, organization, and metacognitive statements as predictors. The pretest scores of motivation were also included as predictor. This regression analysis showed that the posttest motivation scores were predicted by the pretest motivation scores, $\beta=$ $0.52, p<0.01, \Delta R^{2}=0.30$, and by the frequency of statements reflecting personal utility in the learning journals, $\beta=$ $0.44, p<0.01 ; \Delta R^{2}=0.19$. Together, the regression analyses indicate, first, that it was especially the students learning motivation at the end of the intervention that predicted learning outcomes in the comprehension test. Second, the analyses show that the students' post-intervention learning motivation could in fact be traced back to the intensity in which they reflected in their learning journals about the personal relevance of the learning contents.

\section{Discussion}

The aim of the current study was to investigate (1) whether a personal-utility prompt would lead students to reflect about the personal relevance of the learning contents in their learning journals and (2) whether such reflection would increase students' learning motivation as well as their comprehension of the topics. To answer these questions, we conducted a quasi-experimental study with two conditions, a personalutility prompt condition and a standard prompts condition. Students in both conditions received a combination of metacognitive and cognitive prompts that had proved to be successful in previous research $[7,13]$. In the personal-utility prompt condition, students additionally received a prompt that encouraged them to think about the personal relevance of the topic. The results can be summarized as follows.

Students who received a personal-utility prompt included more statements about the meaning, purpose, and relevance of the topic in their learning journal entries than students who did not receive such a prompt (Hypothesis 1). Thus, the students in the personal-utility prompt condition used their learning journals to reflect on their personal stance by self-explaining the relevance of a topic related to their own experiences and personal examples. Students' personalutility statements indicated that they related the learning content, immunology, to real-life situations by discussing its relevance for these situations in their learning journals.

Regarding learning motivation, we found that students who received a personal-utility prompt evaluated journal writing as more valuable to them than was the case for students who received cognitive and metacognitive prompts only. More importantly, as predicted by Hypothesis 2, the students in the personal-utility prompt condition not only evaluated journal writing more positively but also reported a greater motivation for learning biology after the journal writing period as compared with the students in the standard prompts condition. Thus, writing about the meaning, purpose, and relevance of a particular topic had considerable positive effects on students' motivation for studying this topic. The higher posttest motivation scores in the personal-utility prompt condition suggest that, as a result of journal writing, these students became more interested in biological topics, such as immunology, and were more willing to expend effort in learning about this subject. Such improved effort, engagement, and persistence $[16,23,38]$ are important preconditions for meaningful learning [5]. Furthermore, being able to recognize and articulate the personal relevance of a topic also is important for developing a commitment to life-long learning. Life-long learning in general is imperative to the modern knowledgebased society; it is especially important in subject domains where scientific knowledge accumulates and changes rapidly, such as in biology [39]. Accordingly, students who were explicitly prompted to think and write about the personal utility of a biological topic were more likely to engage in the topic with a higher learning motivation.

Reflecting on the personal relevance of the topic not only increased the students' learning motivation but also helped them to better comprehend the learning contents. Accordingly, the students in the personal-utility prompt condition clearly outperformed the students in the standard prompts condition with regard to the level of comprehension (Hypothesis 3). The regression analyses suggest that the students' improved comprehension of immunology was apparently mainly due to the increase in learning motivation, which itself resulted from students' reflecting about the personal relevance of immunology in their learning journals. Hence, a major benefit the students drew from journal writing was motivational. The way they wrote about immunology in the personal-utility prompt condition raised their motivation for learning about this topic and thereby may have also improved their topic-specific learning behavior beyond the journal writing. For example, students may have built a strong intention to understand immunology and consequently increased the application of deep-level comprehension strategies in and out of class. Given that the motivational effects of journal writing apparently reached beyond the learning journal and generally affected students' ways of dealing with the subject, future research should explore how students' learning behavior changes as a result of journal writing. For this purpose, it is necessary to observe students' learning activities related to biology more comprehensively, for example, by assessing and observing students' engagement and strategy use during the lessons as well as during homework and class preparation.

What are the theoretical implications of the present study? Previous research on journal writing concentrated on supporting the application of cognitive and metacognitive strategies in the learning journals in order to foster self-regulated learning $[7,8,10]$. Although this cognitive approach proved to be successful in several short-term laboratory studies, a longitudinal study on journal writing by Nückles et al. [15] found, in the long run, a decrease in students' learning motivation that was associated with a decrease in learning outcomes. Hence, prompting cognitive and metacognitive strategies was not sufficient for maintaining effort and interest in the learning contents over a longer period of time. In the present study with 
relatively young high school students (compared to the university freshmen of [15]), cognitive and metacognitive strategy use in the learning journals was low and did not predict learning outcomes. Nevertheless, prompting reflection about the personal relevance of the learning contents in the learning journals evidently yielded strong effects on learning motivation which was positively related to learning outcomes as measured by a curriculum-based comprehension test. Thus, an important benefit of journal writing has also to be seen in the potential to foster learning motivation, in particular, appreciation of and interest in a topic, and therefore learning outcomes. It is beyond controversy that learning motivation is important for the depth of students' comprehension, but also especially for effort and persistence $[16,38]$. However, learning motivation was not explicitly promoted in previous studies on journal writing. The present study therefore extends this research by providing evidence that learning journals can be used to promote learning motivation as well. The finding that the rather infrequent cognitive and metacognitive strategies in the learning journals of our 7 th grade students did not predict posttest comprehension requires further research. One possible explanation is that thinking about the relevance of a topic could have supported students' deep examination of the topic beyond journal writing. Thus, students could have regarded journal writing as the initiator rather than as the medium for high quality learning. Another possible explanation refers to the competency to apply cognitive and metacognitive strategies in the written texts. The students in our previous studies (see $[7,8,13,15]$ ) were at least 9th graders and often university students. Thus, it is possible that the comparatively younger students in our present study were not able to use the cognitive and metacognitive strategies implied by the prompted strategies to improve their task performance, because they did not possess the necessary cognitive requirements (mediators) to benefit from the strategies. In this case, they would have suffered from a mediation deficiency with regard to the application of cognitive or metacognitive strategies during writing (see $[13,40])$. The low frequency of most types of these strategies (except organizational strategies) in the learning journals, despite the explicit introduction, may support this tentative conclusion.

The empirical results presented in this paper can be easily applied to schools. Previous studies showed that journal writing is a beneficial form of a follow-up course work [7$9,12]$. Results of the present study showed that learning journals are also a promising medium to improve students' learning motivation. Including a personal-utility prompt in the writing instruction invited the students to think about the meaning and purpose of a topic in real-life situations. As a consequence, they were more willing to expend effort in learning biology. As one cannot assume that all students are intrinsically motivated per se and willing to invest substantial effort in exploring a new topic, learning journals offers a promising approach to stimulate and to maintain students' learning motivation.

According to the biology curriculum in German high schools, understanding human biology should help students understand the functioning of their own body and its systemic relations to the social and ecological environment $[2,34]$. This might enable them to act responsibly in order to protect one's own and others' health, for example, with regard to preventing infection with HIV. The relevance of the topic of immunology to students and to their own lives seems to be obvious. Nevertheless, the present study shows that in order to get students to reflect about the personal relevance of this topic, they had to be prompted to do so. Thus, even if the topics to be discussed in the science class are relatively close to the students' realm of experience, it might nevertheless be necessary to support them in reflecting about the value and personal utility of the topics by prompts. As the present study demonstrated, supporting even young students in this way yielded large effects on their learning motivation and thus on their comprehension of the topic.

\section{Appendices}

\section{A. Motivation Questionnaires}

\section{A.1. Journal Acceptance Questionnaire}

(1) Journal writing was useful.

(2) Journal writing was helpful for better understanding of my own way of studying.

(3) Journal writing was helpful to find out what topics I should rework.

(4) Journal writing was helpful for better understanding of the learning contents.

(5) Journal writing was fun for me.

(6) Journal writing was boring. ${ }^{\mathrm{R}}$

(7) Journal writing was beneficial for learning.

(8) I spent low effort in journal writing. ${ }^{\mathrm{R}}$

(9) Journal writing was an important experience for me.

(10) I would like to write learning journals more often as homework.

Note: ${ }^{\mathrm{R}}$ reverse-coded items.

A.2. Intrinsic Motivation Questionnaire. Please think about your class preparation for biology lessons in the last week. How much do you agree with the following statements?

(1) I enjoyed tricky tasks and puzzles in biology very much.

(2) I could solve my tasks pretty good in biology.

(3) I put a lot of effort into preparation of biology lessons.

(4) It was important for me to do well in biology.

(5) I tried hard while solving problems in biology.

(6) Solving problems in biology was fun.

(7) Pretending to be a scientist of biology who explores problems was very interesting. 
TABLE 3: Means, standard deviations, and item difficulties of the comprehension test.

\begin{tabular}{|c|c|c|c|c|c|c|c|}
\hline \multirow{2}{*}{ Item } & \multirow{2}{*}{ Maximum score } & \multicolumn{3}{|c|}{ Pretest } & \multicolumn{3}{|c|}{ Posttest } \\
\hline & & Mean & $\mathrm{SD}$ & ID & Mean & $\mathrm{SD}$ & ID \\
\hline Please explain the differences between HIV and AIDS. & 6 & 1.53 & 0.57 & .26 & 3.59 & 0.83 & .60 \\
\hline $\begin{array}{l}\text { Why is "AIDS" so life threatening for humans? Provide } \\
\text { two reasons. }\end{array}$ & 2 & 0.83 & 0.39 & .41 & 1.79 & 0.62 & .90 \\
\hline $\begin{array}{l}\text { Please name the different components of blood and } \\
\text { their functioning. }\end{array}$ & 6 & 1.68 & 1.03 & .28 & 2.55 & 2.10 & .43 \\
\hline $\begin{array}{l}\text { Put yourself in the position of a red blood cell and run } \\
\text { through the entire circulatory system. Start in the heart } \\
\text { and explain each station you run through. What is } \\
\text { happening to you? }\end{array}$ & 4 & 0.47 & 0.72 & .12 & 1.50 & 1.28 & .38 \\
\hline $\begin{array}{l}\text { Please explain why humans get childhood diseases (e.g., } \\
\text { measles, mumps) only once. }\end{array}$ & 4 & 0.57 & 0.72 & .14 & 2.00 & 1.31 & .50 \\
\hline
\end{tabular}

Note. SD: standard deviation; ID: item difficulty.

(8) I was satisfied with my performance in biology lessons and exams.

(9) I felt tense while performing difficult tasks in bio$\log \mathrm{p}^{\mathrm{R}}$

(10) I felt anxious while solving tasks in biology. ${ }^{\mathrm{R}}$

(11) I felt pressured while doing my biology homework. ${ }^{\mathrm{R}}$

(12) I did not try hard when preparing for biology lessons. ${ }^{\mathrm{R}}$

(13) While preparing for biology, I thought how much I enjoyed it.

(14) I feel pretty competent related to biology tasks.

(15) I was relaxed while preparing biology lessons.

(16) I am proud of my abilities in biology.

(17) The biology tasks did not hold my attention. ${ }^{\mathrm{R}}$

(18) I worried about solving biological tasks. ${ }^{\mathrm{R}}$

Note: ${ }^{R}$ reverse-coded items.

\section{B. Item Statistics for the Comprehension Test}

For more details see Table 3 .

\section{Acknowledgment}

The data reported in this paper were collected by J. Maier as partial fulfillment of the requirements for her teacher degree at the University of Freiburg. All data were completely reanalyzed in preparation for this paper. The authors would like to thank Beatrice Schönmetz and Ruth Deutschländer for their assistance in coding the learning journals. They also wish to thank Wesley Dopkins for his proofreading.

\section{References}

[1] B. J. Zimmerman, "Becoming a self-regulated learner: an overview," Theory into Practice, vol. 41, no. 2, p. 64, 2002.
[2] OECD, Organisation for Economic Cooperation and Development. Programme for International Student Assessment, 2012, http://www.oecd.org/pisa/aboutpisa/.

[3] A. Assor, H. Kaplan, and G. Roth, "Choice is good, but relevance is excellent: autonomy-enhancing and suppressing teacher behaviours predicting students' engagement in schoolwork," British Journal of Educational Psychology, vol. 72, no. 2, pp. 261-278, 2002.

[4] J. R. Kirby and M. J. Lawson, Eds., Enhancing the Quality of Learning: Dispositions, Instruction, and Mental Structures, Cambridge University Press, Cambridge, NY, USA, 2012.

[5] R. E. Mayer, "Rote versus meaningful learning," Theory into Practice, vol. 41, no. 4, pp. 226-232, 2002.

[6] C. Bereiter and M. Scardamalia, "Intentional learning as a goal of instruction," in Knowing, Learning, and Instruction: Essays in Honor of Robert Glaser, L. B. Resnick, Ed., pp. 361-392, Lawrence Erlbaum Associates, Hillsdale, NJ, USA, 1989.

[7] M. Nückles, S. Hübner, and A. Renkl, "Enhancing selfregulated learning by writing learning protocols," Learning and Instruction, vol. 19, no. 3, pp. 259-271, 2009.

[8] K. Berthold, M. Nückles, and A. Renkl, "Do learning protocols support learning strategies and outcomes? The role of cognitive and metacognitive prompts," Learning and Instruction, vol. 17 , no. 5, pp. 564-577, 2007.

[9] A. R. McCrindle and C. A. Christensen, "The impact of learning journals on metacognitive and cognitive processes and learning performance," Learning and Instruction, vol. 5, no. 2, pp. 167-185, 1995.

[10] M. Nückles, S. Hübner, and A. Renkl, "Fostering self-regulated learning by journal writing: how should instructional support be designed to promote high-quality learning?" in Enhancing the Quality of Learning: Dispositions, Instruction, and Learning Processes, J. R. Kirby and M. J. Lawson, Eds., pp. 178-200, Cambridge University Press, New York, NY USA, 2012.

[11] C. M. Reigeluth and F. S. Stein, "The elaboration theory of instruction," in Instructional-Design Theories and Models: An Overview of Their Current Status, C. M. Reigeluth, Ed., pp. 335-382, Erlbaum, Hillsdale, NJ, USA, 1983.

[12] I. Glogger, L. Holzäpfel, R. Schwonke, M. Nückles, and A. Renkl, "Activation of learning strategies in writing learning journals the specificity of prompts matters," Zeitschrift fur Padagogische Psychologie, vol. 23, no. 2, pp. 95-104, 2009.

[13] S. Hübner, M. Nückles, and A. Renkl, "Writing learning journals: instructional support to overcome learning-strategy 
deficits," Learning and Instruction, vol. 20, no. 1, pp. 18-29, 2010.

[14] W. Y. Lan, “The effects of self-monitoring on students' course performance, use of learning strategies, attitude, selfjudgment ability, and knowledge representation," Journal of Experimental Education, vol. 64, no. 2, pp. 101-115, 1996.

[15] M. Nückles, S. Hübner, S. Dümer, and A. Renkl, "Expertise reversal effects in writing-to-learn," Instructional Science, vol. 38, no. 3, pp. 237-258, 2010.

[16] A. Efklides, "Interactions of metacognition with motivation and affect in self-regulated learning: the MASRL model," Educational Psychologist, vol. 46, no. 1, pp. 6-25, 2011.

[17] P. R. Pintrich, "A conceptual framework for assessing motivation and self-regulated learning in college students," Educational Psychology Review, vol. 16, no. 4, pp. 385-407, 2004.

[18] M. Boekaerts, "Self-regulated learning at the junction of cognition and motivation," European Psychologist, vol. 1, no. 2, pp. 100-112, 1996.

[19] P. H. Winne and A. Hadwin, "Studying as self-regulated learning," in Metacognition in Educational Theory and Practice, D. J. Hacker, J. Dunlosky, and A. Graesser, Eds., pp. 277-304, Erlbaum, Hillsdale, NJ, USA, 1998.

[20] B. J. Zimmerman, "Attaining self-regulation: a social cognitive perspective," in Handbook of Self-Regulation, pp. 13-39, Academic Press, San Diego, Calif, US, 2000.

[21] J. S. Eccles and A. Wigfield, "Motivational beliefs, values, and goals," Annual Review of Psychology, vol. 53, pp. 109-132, 2002.

[22] A. Wigfield, J. S. Eccles, R. Roeser, and U. Schiefele, "Development of achievement motivation," in Child and Adolescent Development: An Advanced Course, W. Damon and R. M. Lerner, Eds., pp. 933-1002, John Wiley \& Sons, New Jersey, NJ, USA, 2008.

[23] R. M. Ryan and E. L. Deci, "Intrinsic and extrinsic motivations: classic definitions and new directions," Contemporary Educational Psychology, vol. 25, no. 1, pp. 54-67, 2000.

[24] C. A. Wolters, "Understanding procrastination from a selfregulated learning perspective," Journal of Educational Psychology, vol. 95, no. 1, pp. 179-187, 2003.

[25] C. Tabernero and R. E. Wood, "Interaction between selfefficacy and initial performance in predicting the complexity of task chosen," Psychological Reports, vol. 105, no. 3, pp. 11671180, 2009.

[26] B. J. Zimmerman and A. Bandura, "Impact of self-regulatory influences on writing course attainment," American Educational Research Journal, vol. 31, no. 4, pp. 845-862, 1994.

[27] T. J. Cleary and P. P. Chen, "Self-regulation, motivation, and math achievement in middle school: variations across grade level and math context," Journal of School Psychology, vol. 47, no. 5, pp. 291-314, 2009.

[28] T. Bouffard-Bouchard, "Influence of self-efficacy on performance in a cognitive task," The Journal of Social Psychology, vol. 130, pp. 353-363, 1990.

[29] L. Harlow, T. Debacker, and H. M. Crowson, "Need for closure, achievement goals, and cognitive engagement in high school students," Journal of Educational Research, vol. 104, no. 2, pp. 110-119, 2011.

[30] C. O. Walker, B. A. Greene, and R. A. Mansell, "Identification with academics, intrinsic/extrinsic motivation, and selfefficacy as predictors of cognitive engagement," Learning and Individual Differences, vol. 16, no. 1, pp. 1-12, 2006.

[31] G. V. Caprara, R. Fida, M. Vecchione et al., "Longitudinal analysis of the role of perceived self-efficacy for self-regulated learning in academic continuance and achievement," Journal of Educational Psychology, vol. 100, no. 3, pp. 525-534, 2008.
[32] G. E. McPherson and B. J. Zimmerman, "Self-regulation of musical learning: a social cognitive perspective," in The New Handbook on Music Teaching and Learning, R. Colwell, Ed., pp. 327-347, Oxford University Press, New York, NY, USA, 2002.

[33] M. Boekaerts, "Self-regulated learning: a new concept embraced by researchers, policy makers, educators, teachers, and students," Learning and Instruction, vol. 7, no. 2, pp. 161-186, 1997.

[34] J. A. Ramaley and R. R. Haggett, "Engaged and engaging science: a component of a good liberal education," Peer Review, vol. 7, no. 2, pp. 8-12, 2005.

[35] E. L. Deci and R. M. Ryan, "Intrinsic motivation inventory," 2012, http://www.selfdeterminationtheory.org/.

[36] G. Erkens, G. Kanselaar, M. Prangsma, and J. Jaspers, "Computer support for collaborative and argumentative writing," in Powerful Learning Environments: Unravelling Basic Components and Dimensions, E. de Corte, L. Verschaffel, N. Entwistle, and J. van Merrienboer, Eds., pp. 159-177, Pergamon, Amsterdam, The Netherlands, 2003.

[37] J. Cohen, Statistical Power Analysis for the Behavioral Sciences, Lawrence Erlbaum Associates, Hillsdale, NY, USA, 2nd edition, 1988.

[38] C. M. Chiu and E. T. G. Wang, "Understanding Web-based learning continuance intention: the role of subjective task value," Information and Management, vol. 45, no. 3, pp. 194201, 2008.

[39] S. D. Tunnicliffe and C. Ueckert, "Teaching biology—the great dilemma," Journal of Biological Education, vol. 41, no. 2, pp. 51-52, 2007.

[40] M. Pressley and K. R. Harris, "Cognitive strategies instruction: from basic research to classroom instruction," in Handbook of Educational Psychology, P. A. Alexander and P. H. Winne, Eds., pp. 265-286, Lawrence Erlbaum Associates Publishers, Mahwah, NJ, USA, 2006. 


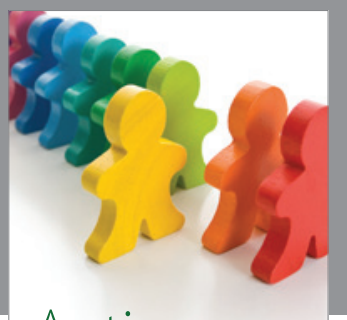

Autism

Research and Treatment
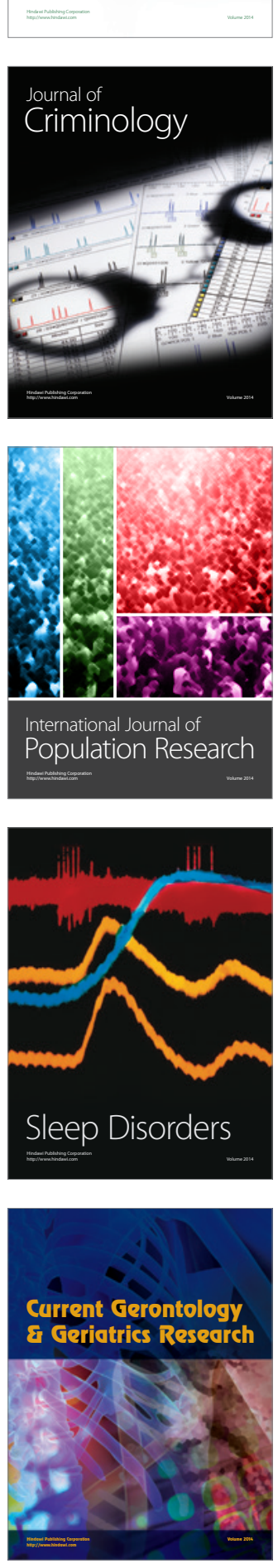
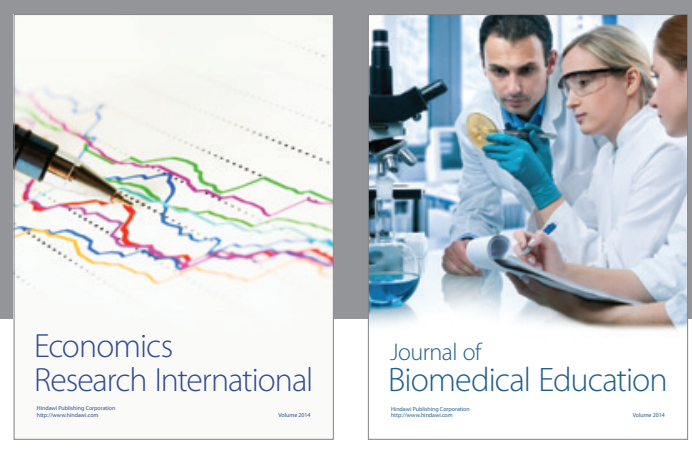

Journal of

Biomedical Education

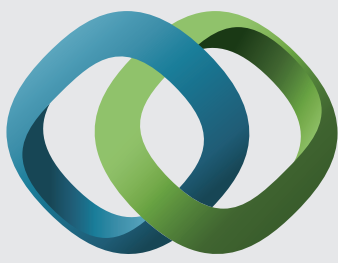

\section{Hindawi}

Submit your manuscripts at

http://www.hindawi.com
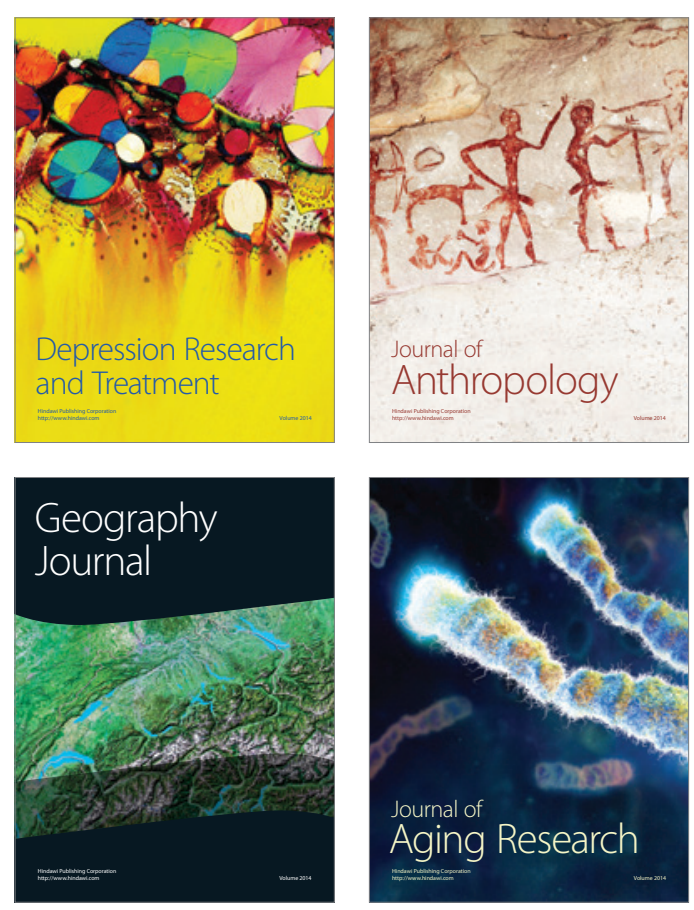

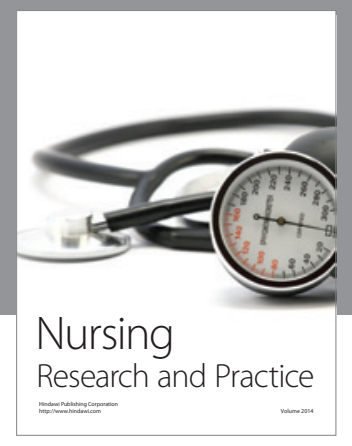

Nursing

Research and Practice

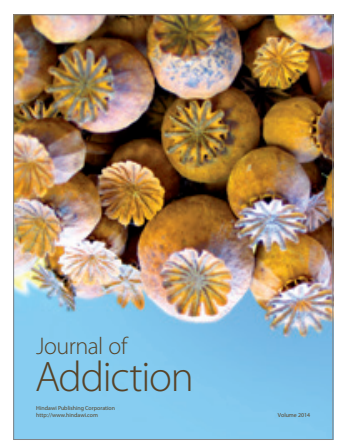

Child Development

Research

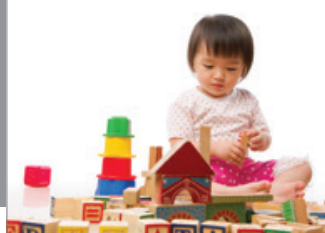

迥
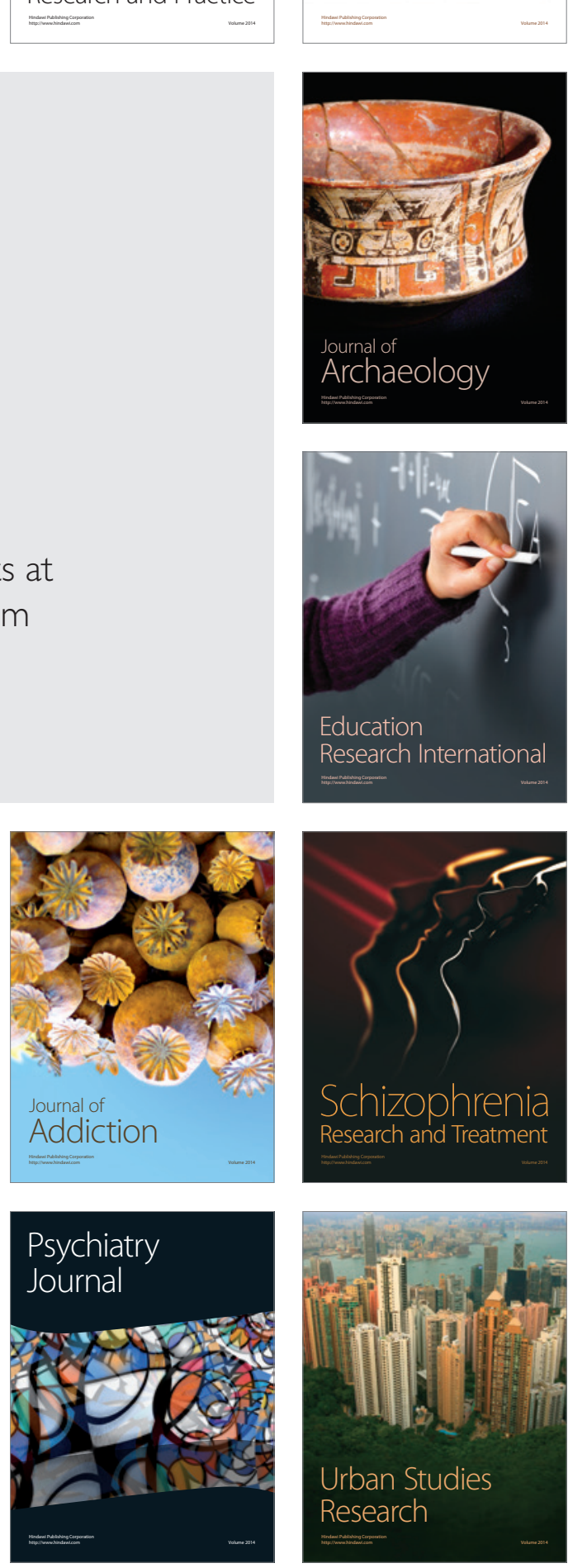